\title{
Preparation and Characterization of some Transition Metal Complexes with Crotyl xanthate Ligand and their Adducts with Nitrogen Bases
}

\author{
Saad E. Al- Mukhtar Hiba F. Al-Katib Lana A. Al-Nuaimy \\ Department of Chemistry/ College of Science/ University of Mosul
}

(Received 9/6/2013;Accepted 7/10/2013)

\begin{abstract}
A new series of xanthate complexes of the general formula [M(Crotxant $\left.)_{2}\right],\left[\mathrm{M}(\text { Crotxant })_{2} \cdot \mathrm{nL}\right]$ where $\mathrm{M}=\mathrm{Fe}(\mathrm{II}), \mathrm{Co}(\mathrm{II}), \mathrm{Ni}(\mathrm{II}), \mathrm{Cu}(\mathrm{II})$, Crotxant $=$ Crotyl xanthate, $\mathrm{n}=2, \mathrm{~L}=$ pyridine, quinoline, 4-picoline, $\mathrm{n}=1, \mathrm{~L}=$ ethylenediamin , 1,10- phenanthroline. All the isolated complexes have been prepared and characterized by atomic absorption, molar conductivity, Infrared, electronic absorption spectra and magnetic measurement.

The conductivity measurements proved the non-electrolytic behaviour of all compounds, magnetic susceptibility measurements and electronic spectra show a tetrahedral geometry for complexes of the type $\left[\mathrm{M}(\mathrm{Crotxant})_{2}\right]$ and octahedral geometry for complexes of the type $\left[\mathrm{M}(\text { Crotxant })_{2} \cdot \mathrm{nL}\right]$.
\end{abstract}

Keywords: xanthate, Nickel(II), Cobalt(II), Copper(II), Iron(II).

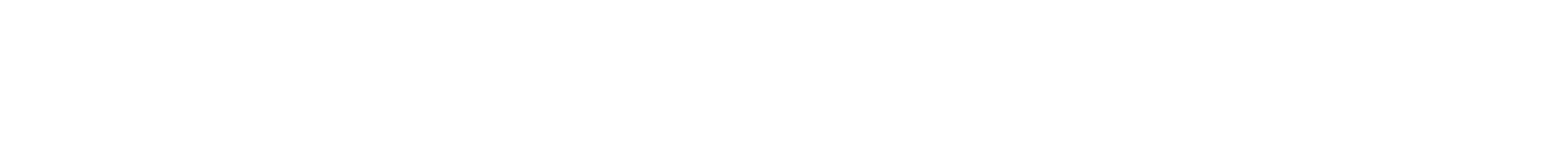

\section{الملغص}

حضرت معقدات جدية للزالثيت ذات الصيغة [M(Crotxant)2.nL] ، [MCrotxant) حيث أن:

M=Fe(II), Co(II), Ni(II) and Cu(II) Crotxant = كروتايل زانثيت

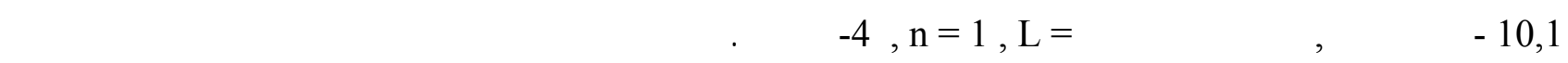

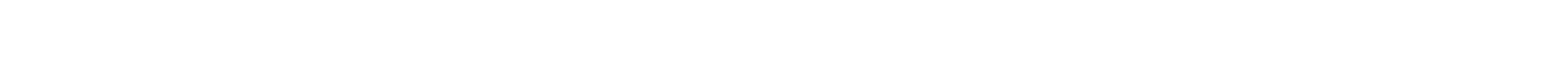
التوصيلية المولارية على السلوك غير الالكتروليتي لجمبع المعددات ودلت القيلست المغنطيسية والطيفيف الالكتروني ـة على

التخاذ المعداتشكل رباعي للنطوح للصيغة [M(Crotxant)

\section{INTRODUCTION}

Xanthates and related ligands as well as transition-metal complexes in which a 1,1-dithiolate ligand forms a four-membered ring with the metal ion have been extensively investigated (Coucouvanis, 1970). 1,1- dithiolate ligands found many applications ranging from flotation agents to radical polymerization (Haiduc, 2007; Coote and Radom, 2004; Wan et al., 2005; Tiekink and Haiduc, 2005). Xanthate ligands $\left[\mathrm{ROCS}_{2}\right]$ are known to coordinate metal centers in a variety of coordination modes, e.g. monodentate, bidentate chelating or bridging (Haiduc, 2003). The ligands can be easily prepared and their properties can be suitably modified by the appropriate choice of the $\mathrm{O}$ bound substituents. Further, the metal xanthate complexes show that the interesting thermal behaviour and thermal decomposition of such complexes can be a useful route to metal sulfides (Hill et al., 1994). Recently, metal alkylxanthate compounds have been used as synthetic precursors for the generation of metal sulfide nanostructures (Bessergenev et al., 1995; Bessergenev et al., 
1998; Pradhan et al., 2003). In view of this, we decided to explore the suitability of crotyl xanthates complexes with $\mathrm{Fe}(\mathrm{II}), \mathrm{Co}(\mathrm{II}), \mathrm{Ni}(\mathrm{II})$ and $\mathrm{Cu}(\mathrm{II})$ as single-source precursors for the preparation of nanocrystalline transition metal sulfide.

\section{Materials and instrumentation}

\section{EXPERIMENTAL}

The reagents and solvents were of analytical grade. (1,10)- Phenanthroline, crotyl alcohol, carbon disulfide were purchased from Merck Company,pyridine,4-picoline,quinoline were purchased from BDH company. All melting points were determined on a Gallen Kamp and Electro thermal 9300 Digital-Series apparatus were uncorrected. The IR. - Spectra using (KBr disc) were recorded on Perkin - Elmer 590B Spectrophotometer. UV -Visible spectra were recorded using a Shimadzu UV-160 spectrophotometer for $10^{-3} \mathrm{M}$ solution of complexes in DMF as solvent, the magnetic susceptibilities were measured at $25^{\circ} \mathrm{C}$ on the solid by the Faraday method using Brucker BM6 instrument. Diamagnetic corrections were calculated using Pascal's constants. Conductivities were measured with a Conductivity Hard-Held Meter LF 330 (WTW GmbH) at $25{ }^{\circ} \mathrm{C}$. Metal\% was determined using atomic absorption elemental Analyzer Model 1106.

\section{Synthesis of potassium crotylxanthate ( K Crotxant ) ( Vogel, 2008 )}

To a solution of $(5.61 \mathrm{~g}, 0.1 \mathrm{~mol})$ potassium hydroxide in water, $(7.20 \mathrm{~g}, 0.1 \mathrm{~mol})$ of crotyl alcohol was added with stirring. The mixture was cooled in an ice-bath, to this mixture carbon disulfide $(7.60 \mathrm{~g}, 0.1 \mathrm{~mol})$ was added dropwise with a continuous stirring for $30 \mathrm{~min}$. in the icebath, then allowed to reach room temperature and diethyl ether $\left(50 \mathrm{~cm}^{3}\right)$ was added. The yellow precipitated formed was filtered off washed with ether and dried under vacuum.

\section{Synthesis of Complexes}

\section{A. Synthesis of [M (Crotxant) $\left.)_{2}\right]$}

$\mathrm{M}=\mathrm{Fe}(\mathrm{II}), \mathrm{Co}(\mathrm{II}), \mathrm{Ni}(\mathrm{II}), \mathrm{Cu}(\mathrm{II})$

A solution of (K Crotxant) $(0.002 \mathrm{~mol})$ in $50 \%$ ethanol was added to ethanolic solution of $\mathrm{FeCl}_{2} \cdot 4 \mathrm{H}_{2} \mathrm{O}(0.198,0.001 \mathrm{~mol}), \mathrm{CoCl}_{2} \cdot 6 \mathrm{H}_{2} \mathrm{O}(0.237 \mathrm{~g}, 0.001 \mathrm{~mol}), \mathrm{NiCl}_{2} \cdot 6 \mathrm{H}_{2} \mathrm{O}(0.237 \mathrm{~g}, 0.001 \mathrm{~mol})$ or $\mathrm{CuCl}_{2} .2 \mathrm{H}_{2} \mathrm{O}(0.170 \mathrm{~g}, 0.001 \mathrm{~mol})$ dropwise with constant stirring at room temperature. The green precipitate formed was filtered off and washed with ethanol then with diethyl ether and dried under vacuum.

\section{B. Synthesis of $\left[M(\text { Crotxant })_{2} L_{2}\right]$}

$\mathrm{L}=$ Pyridine, isoquinoline, 4-picoline

These Complexes were prepared similarly as in (A) The precipitate formed was treated with $(0.002 \mathrm{~mol})$ of (Pyridine, isoquinoline, 4-picoline) dropwise with continuous stirring for $30 \mathrm{~min}$, the precipitate formed, was filtered and washed with ethanol then dried under vacuum.

\section{Synthesis of complex [M (Crotxant $\left.)_{2}(\mathrm{~L})\right]$}

$\mathrm{L}=1,10$-phenanthroline, ethylene diamine

Prepared similarly as in (A ). The precipitate formed was treated with $(0.001 \mathrm{~mol})$ of $(1,10-$ phenanthroline, ethylenediamine) dropwise with continuous stirring for $30 \mathrm{~min}$, the precipitate formed was filtered, washed with ethanol, then dried under vacuum.

\section{RESULTS AND DISCUSSION}

The new ligand was prepared by the reaction of Crotyl alcohol with potassium hydroxide and carbon disulphide. The complexes were prepared through the direct reaction of the metal chlorides, $\mathrm{FeCl}_{2} \cdot 4 \mathrm{H}_{2} \mathrm{O}, \mathrm{CoCl}_{2} \cdot 6 \mathrm{H}_{2} \mathrm{O}, \mathrm{NiCl}_{2} \cdot 6 \mathrm{H}_{2} \mathrm{O}$, or $\mathrm{CuCl}_{2} .2 \mathrm{H}_{2} \mathrm{O}$ with the above ligand in $(1: 2)$ molar ratio. The values of molar conductivity in dimethyl formamide solution of the complexes range from $(2.1-39) \Omega^{-1} . \mathrm{cm}^{2} . \mathrm{mol}^{-1}$, which are within the values for the non electrolyte type (Geary, 1971). 


\section{Infrared spectral studies}

The important IR bands of the ligand and its complexes are listed in Table (2). The v(C - O) and $v(C-S)$ were observed in the ranges of $(1147-1211) \mathrm{cm}^{-1}$ and $(940-1050) \mathrm{cm}^{-1}$ respectively. The presence of only one band in the later rëgion reports the bidentate coordination of the dithio ligand (Serrano, 2003).

IR spectra showed a new band at $(408-445) \mathrm{cm}^{-1}$ which can be considered as an evidence for the coordination of metal to sulfur v(M-S). (Raya et al., 2006)

\section{Electronic spectral studies}

The UV-Visible spectra of the complexes in $10^{-3} \mathrm{M}$ solution DMF are recorded; the results are listed in Table (2). The Fe(II) complex (1) give an absorption band at $\left(9523 \mathrm{~cm}^{-1}\right)$, which corresponds to $\left({ }^{5} \mathrm{E} \rightarrow{ }^{5} \mathrm{~T}_{2}\right)$ transition in a tetrahedral geometry (Nicholls, 1973). Complexes (2-6) show absorption bands at the range (9803-10964) $\mathrm{cm}^{-1}$, which were assigned to $\left({ }^{5} \mathrm{~T}_{2} \mathrm{~g} \rightarrow{ }^{5} \mathrm{Eg}\right)$ transition in an octahedral geometry (Coucouvanis and Fackler, 1967).

The Co(II) complex (7) exhibited two absorption bands at $\left(10093 \mathrm{~cm}^{-1}\right)$ and $\left(13921 \mathrm{~cm}^{-1}\right)$ which were assigned to $\left({ }^{4} \mathrm{~A}_{2}(\mathrm{~F}) \rightarrow{ }^{4} \mathrm{~T}_{1}(\mathrm{~F}),\left({ }^{4} \mathrm{~A}_{2}(\mathrm{~F}) \rightarrow{ }^{4} \mathrm{~T}_{1}(\mathrm{P})\right)\right.$ transitions respectively (Siddiqi and Nishat, $2000)$, in tetrahedral configuration of this complex. The $\left({ }^{4} \mathrm{~A}_{2}(\mathrm{~F}) \rightarrow{ }^{4} \mathrm{~T}_{2}(\mathrm{~F})\right)$ transition is out of the range of the instrument used. Complexes (8-12) show three absorption bands at the range (10183$\left.10692 \mathrm{~cm}^{-1}\right), \quad\left(13009-15069 \mathrm{~cm}^{-1}\right)$ and $\left(19064-21432 \mathrm{~cm}^{-1}\right)$, which were assigned to $\left({ }^{4} \mathrm{~T}_{1} \mathrm{~g}(\mathrm{~F}) \rightarrow{ }^{4} \mathrm{~T}_{2} \mathrm{~g}(\mathrm{~F})\right),\left({ }^{4} \mathrm{~T}_{1} \mathrm{~g}(\mathrm{~F}) \rightarrow{ }^{4} \mathrm{~A}_{2} \mathrm{~g}(\mathrm{~F})\right)$ and $\left({ }^{4} \mathrm{~T}_{1} \mathrm{~g}(\mathrm{~F}) \rightarrow{ }^{4} \mathrm{~T}_{1} \mathrm{~g}(\mathrm{P})\right)$ transitions in an octahedral geometry (Martel, 1971).

The Ni(II) complex (13) show two absorption bands at $\left(10224 \mathrm{~cm}^{-1}\right),\left(13572 \mathrm{~cm}^{-1}\right)$ which were assigned to $\left({ }^{3} \mathrm{~T}_{1}(\mathrm{~F}) \rightarrow{ }^{3} \mathrm{~A}_{2}(\mathrm{~F})\right)$ and $\left({ }^{3} \mathrm{~T}_{1}(\mathrm{~F}) \rightarrow{ }^{3} \mathrm{~T}_{1}(\mathrm{P})\right)$ transitions in a tetrahedral geometry ( Nicholls, 1973), respectively, the complexes (14-18) show three absorption bands in the range (10157-11806 $\left.\mathrm{cm}^{-1}\right),\left(15726-17921 \mathrm{~cm}^{-1}\right)$ and $\left(19305-20765 \mathrm{~cm}^{-1}\right)$, which were assigned to $\left({ }^{3} \mathrm{~A}_{2} \mathrm{~g}(\mathrm{~F}) \rightarrow{ }^{3} \mathrm{~T}_{2} \mathrm{~g}(\mathrm{~F})\right)$, $\left({ }^{3} \mathrm{~A}_{2} \mathrm{~g}(\mathrm{~F}) \rightarrow{ }^{3} \mathrm{~T}_{1} \mathrm{~g}(\mathrm{~F})\right)$ and $\left({ }^{3} \mathrm{~A}_{2} \mathrm{~g}(\mathrm{~F}) \rightarrow{ }^{3} \mathrm{~T}_{1} \mathrm{~g}(\mathrm{P})\right)$ transition in an octahedral geometry (Singh et al., 1989).

The $\mathrm{Cu}(\mathrm{II})$ complex (19) give an absorption band at $\left(96682 \mathrm{~cm}^{-1}\right)$, which corresponds to $\left({ }^{2} \mathrm{~T}_{2} \rightarrow{ }^{2} \mathrm{E}\right)$ transition in tetrahedral geometry (Cookson et al., 2010), the complexes (20-24) show a broad band in the region $\left(12121-12500 \mathrm{~cm}^{-1}\right)$, which was assigned to $\left({ }^{2} \mathrm{Eg} \rightarrow{ }^{2} \mathrm{~T}_{2} \mathrm{~g}\right)$ transition which may be formed from the combination of three transitions $\left({ }^{2} \mathrm{~B}_{1} \mathrm{~g} \rightarrow{ }^{2} \mathrm{~A}_{1} \mathrm{~g}\right),\left({ }^{2} \mathrm{~B}_{1} \mathrm{~g} \rightarrow{ }^{2} \mathrm{~B}_{2} \mathrm{~g}\right)$ and $\left({ }^{2} \mathrm{~B}_{1} \mathrm{~g} \rightarrow{ }^{2} \mathrm{Eg}\right)$ in an octahedral configuration (Lever et al.,1984).

\section{Magnetic susceptibility measurements}

The magnetic moments of the complexes Table (1) were measured at $\left(25^{\circ} \mathrm{C}\right)$. The magnetic moments for $\mathrm{Fe}(\mathrm{II}), \mathrm{Co}(\mathrm{II}), \mathrm{Ni}(\mathrm{II})$ and $\mathrm{Cu}(\mathrm{II})$ complexes $(1,7,13$ and 19) are in the range (2.255.2B.M) suggest a tetrahedral geometry . The high value for complex (7) is due to second order orbital contribution. Finally the magnetic moment values of the other complexes $(2-6,8-12,14$ $18,20-24)$ were in the range (1.70-5.70 B.M.) suggesting an octahedral geometry (Nicholls, 1973). 
Table 1: Analytical and some physical properties of the prepared complexes.

\begin{tabular}{|c|c|c|c|c|c|c|}
\hline No. & compound & $\begin{array}{c}\text { Decomposition } \\
\text { Temp. ( ( } \mathrm{C} \text { ) }\end{array}$ & Color & $\frac{\Lambda_{\mathrm{M}}}{\mathrm{cm}^{2} \cdot \mathrm{ohm}^{-1} \cdot \mathrm{mol}^{-1}}$ & $\begin{array}{c}\mu_{\text {eff }} \\
\text { B.M } \\
\left(\mathbf{2 5}^{\circ} \mathbf{C}\right)\end{array}$ & $\begin{array}{c}\% \\
\text { Calculated } \\
\text { (Metal } \\
\text { found) } \\
\end{array}$ \\
\hline $\mathrm{L}$ & $\mathrm{K}$ (Crotxant) & $>290$ & yellow & - & & \\
\hline 1. & {$\left[\mathrm{Fe}(\text { Crotxant })_{2}\right]$} & 260 & Yellowish red & 2.1 & 4.99 & $16.19(15.8)$ \\
\hline 2. & {$\left[\mathrm{Fe}(\text { Crotxant })_{2}(\mathrm{py})_{2}\right]$} & 210 & Dark yellow & 9.8 & 4.89 & $9.33(10.2)$ \\
\hline 3. & {$\left[\mathrm{Fe}(\text { Crotxant })_{2}(\text { qui })_{2}\right]$} & 185 & Yellowish red & 13.28 & 4.94 & $10.58(11.34)$ \\
\hline 4. & {$\left[\mathrm{Fe}(\text { Crotxant })_{2}(4-\mathrm{pic})_{2}\right]$} & 185 & Yellowish red & 16.0 & 4.50 & $11.16(11.78)$ \\
\hline 5. & {$\left[\mathrm{Fe}(\text { Crotxant })_{2} \mathrm{en}\right]$} & $>300$ & Dark yellow & 18.8 & 4.98 & $13.89(14.56)$ \\
\hline 6. & {$\left[\mathrm{Fe}(\text { Crotxant })_{2} 1,10\right.$ phen $]$} & 210 & Brown & 9.64 & 5.1 & $10.69(9.94)$ \\
\hline 7. & {$\left[\right.$ Co $\left.(\text { Crotxant })_{2}\right]$} & 258 & Violet & 17.5 & 5.2 & $16.69(16.5)$ \\
\hline 8. & $\left.\left[\mathrm{Co}(\text { Crotxant })_{2}\right)_{2}(\text { py })_{2}\right]$ & $>250$ & Brown & 18.0 & 5.7 & $11.52(11.14)$ \\
\hline 9. & {$\left[\mathrm{Co}(\text { Crotxant })_{2}(\text { qui })_{2}\right]$} & 148 & Dark brown & 18.4 & 5.6 & $9.64(9.5)$ \\
\hline 10. & {$\left[\mathrm{Co}(\text { Crotxant })_{2}(4-\text { pic })_{2}\right]$} & 246 & Light violet & 21.5 & 5.3 & $10.92(9.74)$ \\
\hline 11. & {$\left[\mathrm{Co}(\text { Crotxant })_{2} \mathrm{en}\right]$} & 110 & Brown & 20 & 5.10 & $5.8(6.25)$ \\
\hline 12 . & $\begin{array}{l}{[\text { Co }} \\
\left.(\text { Crotxant })_{2} 1,10 \text { phen }\right]\end{array}$ & 140 & Dark brown & 4.8 & 4.03 & $11.04(10.88)$ \\
\hline 13. & {$\left[\mathrm{Ni}(\text { Crotxant })_{2}\right]$} & 270 & Green & 39 & 2.71 & $16.61(15.0)$ \\
\hline 14. & {$\left[\mathrm{Ni}(\text { Crotxant })_{2}(\mathrm{py})_{2}\right]$} & 145 & Green & 12.8 & 2.93 & $11.47(10.6)$ \\
\hline 15 & {$\left[\mathrm{Ni}(\text { Crotxant })_{2}(\text { qui })_{2}\right]$} & 150 & Gray & 14.2 & 3.81 & $9.59(10.25)$ \\
\hline 16. & {$\left[\mathrm{Ni}(\text { Crotxant })_{2}(4-\text { pic })_{2}\right]$} & $100^{*}$ & Black & 20 & 3.3 & $10.88(9.98)$ \\
\hline 17. & {$\left[\mathrm{Ni}(\text { Crotxant })_{2} \mathrm{en}\right]$} & 110 & Pink & 20.1 & 3.36 & $14.27(15.42)$ \\
\hline 18. & $\begin{array}{l}{[\mathrm{Ni}} \\
\left.(\text { Crotxant })_{2} 1,10 \text { phen }\right]\end{array}$ & 150 & Dark brown & 14.7 & 2.78 & $10.99(10.91)$ \\
\hline 19. & {$\left[\mathrm{Cu}(\text { Crotxant })_{2}\right]$} & 138 & Green & 20 & 2.25 & $17.76(16.67)$ \\
\hline 20 & {$\left[\mathrm{Cu}(\text { Crotxant })_{2}(\text { py })_{2}\right]$} & 148 & Green & 5.5 & 2.30 & $12.31(12.66)$ \\
\hline 21. & {$\left[\mathrm{Cu}(\text { Crotxant })_{2}(\text { qui })_{2}\right]$} & 145 & Green & 10.0 & 2.05 & $10.31(10.5)$ \\
\hline 22. & {$\left[\mathrm{Cu}(\text { Crotxant })_{2}(4-\mathrm{pic})_{2}\right]$} & 150 & Black & 21.6 & 2.18 & 11.68(11.53) \\
\hline 23. & {$\left[\mathrm{Cu}(\text { Crotxant })_{2} \mathrm{en}\right]$} & 110 & Green & 17.5 & 1.70 & $15.28(15.33)$ \\
\hline 24 & $\begin{array}{l}{[\mathrm{Cu}} \\
\left.(\text { Crotxant })_{2} 1,10 \text { phen }\right]\end{array}$ & 150 & Dark Green & 8.0 & 2.09 & $11.81(12.1)$ \\
\hline
\end{tabular}


Table 2: Selected IR bands $\left(\mathrm{cm}^{-1}\right)$ and electronic spectral data of the ligand and it is metal complexes

\begin{tabular}{|c|c|c|c|c|c|}
\hline No. & compound & $v(C-S)$ & $v(C-O)$ & $v(\mathbf{M}-\mathbf{S})$ & $\begin{array}{c}\text { d-d absorption } \\
\mathrm{cm}^{-1}\end{array}$ \\
\hline $\mathrm{L}$ & $\mathrm{K}$ (Crotxant) & 1050 & 1147 & - & - \\
\hline 1. & {$\left[\mathrm{Fe}(\text { Crotxant })_{2}\right]$} & 1022 & 1180 & 445 & 9523 \\
\hline 2. & {$\left[\mathrm{Fe}(\mathrm{Crotxant})_{2}(\mathrm{py})_{2}\right]$} & 1020 & 1184 & 420 & 9803 \\
\hline 3. & {$\left[\mathrm{Fe}(\text { Crotxant })_{2}(\text { qui })_{2}\right]$} & 1028 & 1184 & 420 & 10207 \\
\hline 4. & {$\left[\mathrm{Fe}(\text { Crotxant })_{2}(4-\mathrm{pic})_{2}\right]$} & 1020 & 1186 & 418 & 9833 \\
\hline 5. & {$\left[\mathrm{Fe}(\text { Crotxant })_{2} \mathrm{en}\right]$} & 1022 & 1188 & 426 & 10964 \\
\hline 6. & {$\left[\mathrm{Fe}(\text { Crotxant })_{2} 1,10\right.$ phen $]$} & 1024 & 1170 & 420 & 10917 \\
\hline 7. & {$\left[\right.$ Co $\left.(\text { Crotxant })_{2}\right]$} & 964 & 1207 & 415 & 10093,13921 \\
\hline 8. & {$\left[\operatorname{Co}(\text { Crotxant })_{2}(\text { py })_{2}\right]$} & 1041 & 1178 & 410 & $10325,13009,19064$ \\
\hline 9. & {$\left[\mathrm{Co}(\text { Crotxant })_{2}(\text { qui })_{2}\right]$} & 1022 & 1165 & 408 & $10224,13578,21432$ \\
\hline 10. & {$\left[\mathrm{Co}(\text { Crotxant })_{2}(4-\text { pic })_{2}\right]$} & 1028 & 1161 & 410 & $10692,15069,19982$ \\
\hline 11. & {$\left[\right.$ Co $\left.(\text { Crotxant })_{2} \mathrm{en}\right]$} & 1024 & 1178 & 409 & $10459,14783,20759$ \\
\hline 12 & {$\left[\right.$ Co $(\text { Crotxant })_{2} 1,10$ phen $]$} & 1041 & 1170 & 410 & $10183,13783,20865$ \\
\hline 13. & {$\left[\mathrm{Ni}(\text { Crotxant })_{2}\right]$} & 940 & 1200 & 440 & 10224,13572 \\
\hline 14. & {$\left[\mathrm{Ni}(\text { Crotxant })_{2}(\text { py })_{2}\right]$} & 964 & 1194 & 415 & $10163,17921,19305$ \\
\hline 15. & $[\mathrm{Ni} \text { (Crotxant) })_{2}$ (qui) $\left.)_{2}\right]$ & 960 & 1160 & 438 & $10157,16348,20364$ \\
\hline 16. & {$\left[\mathrm{Ni}(\text { Crotxant })_{2}(4-\text { pic })_{2}\right]$} & 1024 & 1184 & 435 & $11806,15726,20765$ \\
\hline 17. & $\left.[\mathrm{Ni} \text { (Crotxant })_{2} \mathrm{en}\right]$ & 1027 & 1153 & 430 & $10876,17301,20000$ \\
\hline 18. & [Ni (Crotxant) $)_{2} 1,10$ phen] & 1022 & 1180 & 428 & $11625,17587,20509$ \\
\hline 19. & {$\left[\mathrm{Cu}(\text { Crotxant })_{2}\right]$} & 966 & 1188 & 420 & 96682 \\
\hline 20. & {$\left[\mathrm{Cu}(\text { Crotxant })_{2}(\text { py })_{2}\right]$} & 1022 & 1209 & 415 & 12121 \\
\hline 21. & {$\left[\mathrm{Cu}(\text { Crotxant })_{2}(\text { qui })_{2}\right]$} & 1026 & 1200 & 415 & 12290 \\
\hline 22. & {$\left[\mathrm{Cu}(\text { Crotxant })_{2}(4-\text { pic })_{2}\right]$} & 980 & 1211 & 418 & 12376 \\
\hline 23. & {$\left[\mathrm{Cu}(\text { Crotxant })_{2} \mathrm{en}\right]$} & 1022 & 1155 & 410 & 12500 \\
\hline 24. & $[\mathrm{Cu} \text { (Crotxant) })_{2} 1,10$ phen $]$ & 1028 & 1161 & 412 & 12476 \\
\hline
\end{tabular}

melting point $\mathbf{H}^{*}$ 


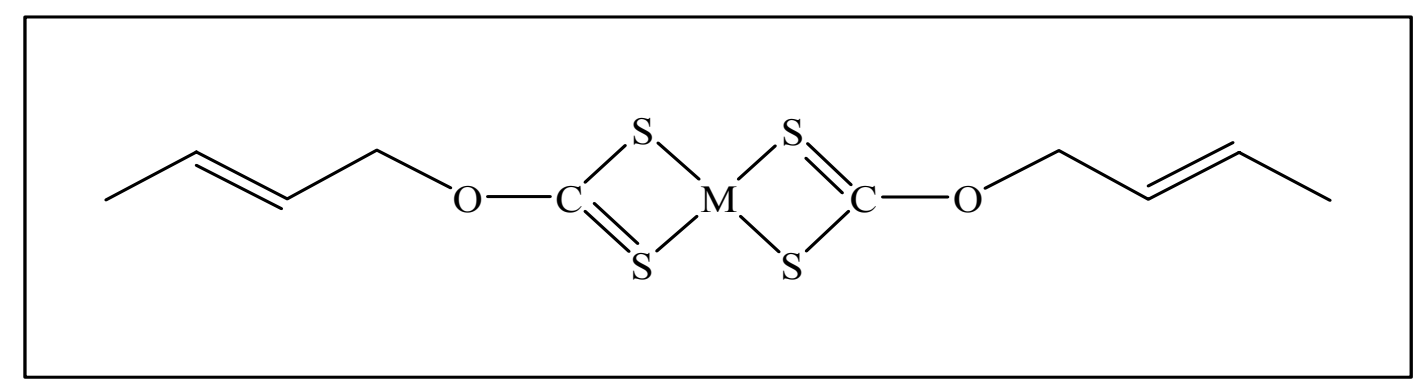

$\mathrm{a}$
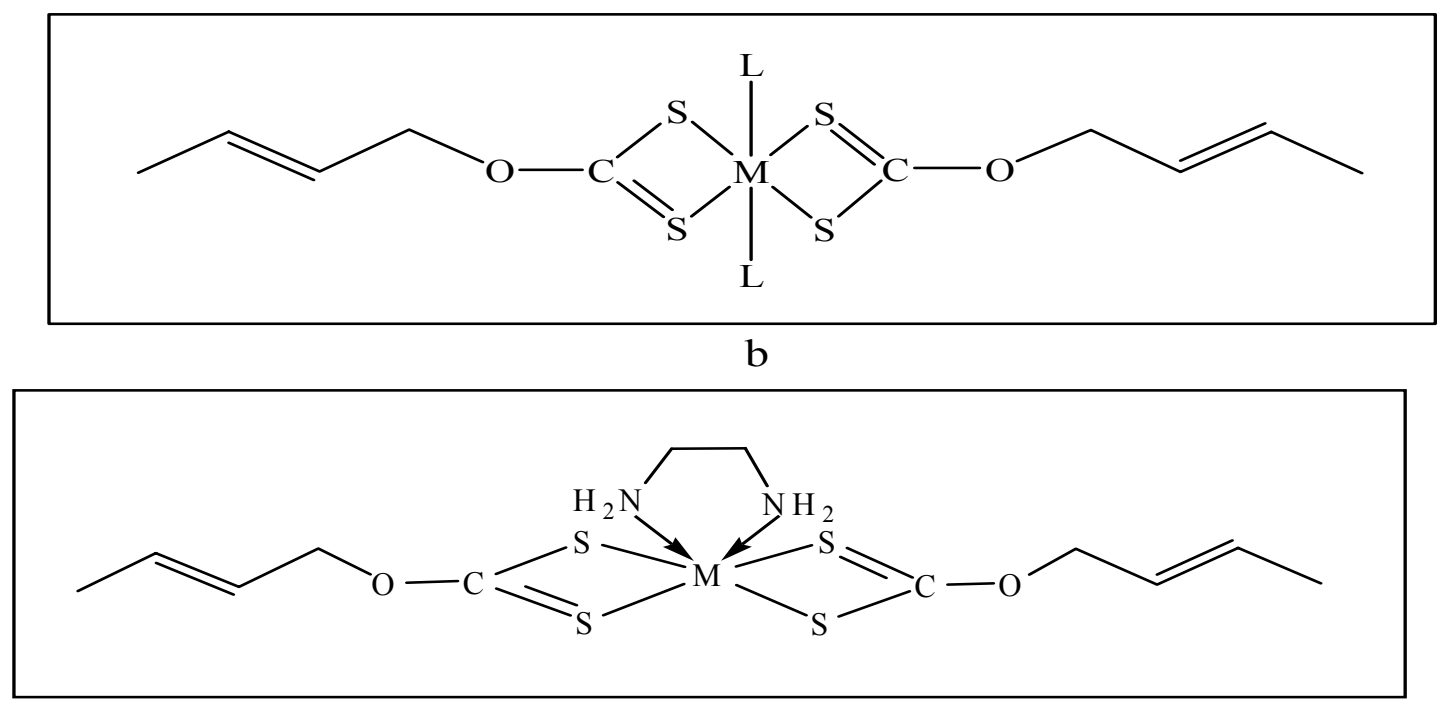

$\mathrm{c}$

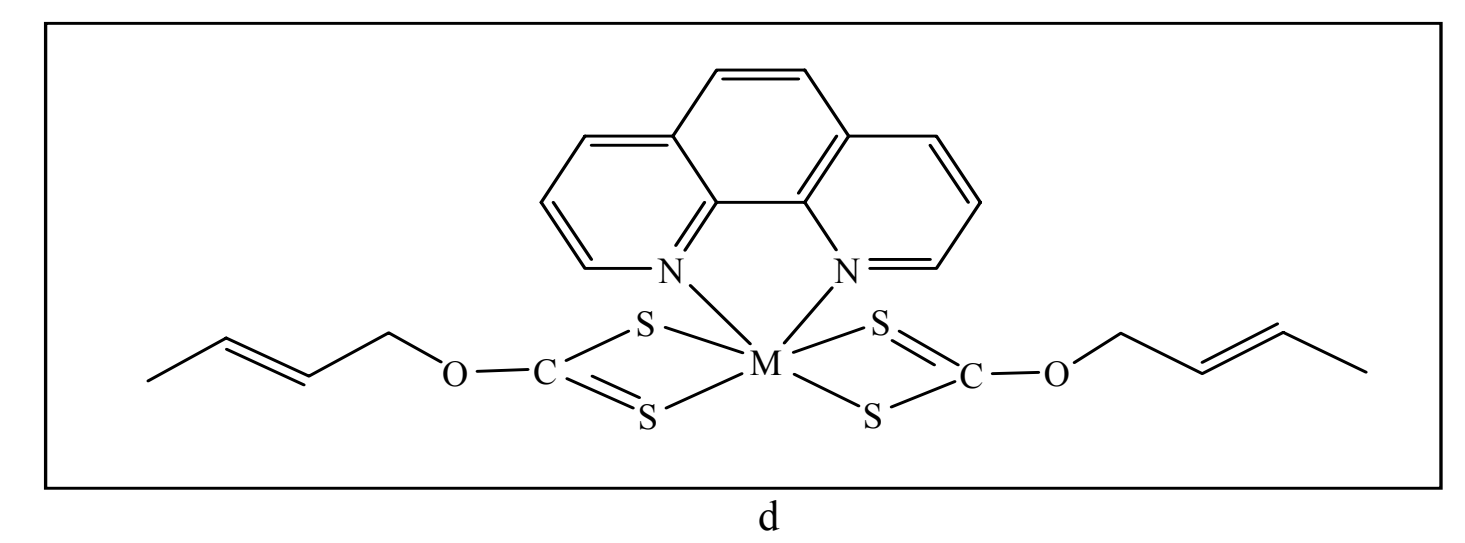

Fig. : Suggested structures (a) complexes $[1,7,13,19]$,

(b) complexes $[2-4,8-10,14-16,20-22] \mathrm{L}=p y$, qui., 4-pic.

(c) complexes $[5,11,17,23]$,

(d) complexes $[6,12,18,24]$

\section{REFERENCES}

Bessergenev, V.G.; Ivanova, E.N.; Kovalevskaya, Yu.A.; Gromilov, S.A.; Kirichenko, V.N.; Zemskova, S.M.; Vasilieva, I.G.; Ayupov, B.M.; Shwarz, N.L. (1995). Optical and structural proper tees of ZnS and ZnS: Mn films prepared by CVD method citations. Mater. Res. Bull., 30(11), 1393-1400.

Bessergenev, V.G.; Bessergenev, A.V.; Ivanova, E.N.; Kovalevskaya, Yu. A. (1998). Study of $\operatorname{In}_{2} \mathrm{~S}_{3}$ thin films by diffraction of synchrotron Radiation. J. Solid State Chem. 137, 6-11. 
Cookson, J.; Emma. A.L.E.; John, P.M.; Christopher, J.S.; Rowena, L.P.; Andrew, R.C.; Michael, G.B.D.; Paul, D. (2010). Metal directed assembly of large dinuclear copper(II) dithiocarbamate Macrocyclic complexes. Inorg Chim Acta., 363, 1195-1203.

Coote, M.L.; Radom, L. (2004). Substituent effects in xanthate-mediated polymerization of vinyl acetate: ab initio evidence for an alternative fragmentation pathway. Macromolecules, 37, 590-596.

Coucouvanis, D. (1970). The chemistry of the dithioacid and 1,1- dithiolate complexes. Prog. Inorg. Chem., 11, 233.

Coucouvanis, D.; Fackler, J.P. (1967). Square-planar sulfur complexes. VI. reactions of bases with xanthate, dithiocarbamates, and dithiolates of nickel(II). Inorg. Chem., 6, 2047.

Geary, W.J. (1971). The use of conductivity measurements in organic solvents for characterization of coordination compounds. Coord. Chem. Rev.7,8.

Haiduc, I. (2007). "Handbook of Chalcogen Chemistry. In: Devillanova F (ed) New Perspectives in Sulfur, Selenium, and Tellurium". RSC Publishing, London, 593 p.

Haiduc, I. (2003). "1,1-Dithiolato Ligands Comprehensive Coordination Chemistry II. from Biology to Nanotechnology". Elsevier, Vol. 1, pp. 349-376.

Hill, J.O.; Murray, J.P.; Patil, K.C. (1994). The thermochemistry of the metal dithiocarbamate and xanthate complexes . Rev. Inorg. Chem., 14, 363 - 387.

Lever, A.B.P. (1984). "Inorganic Electronic Spectroscopy". 2nd ed., Elsevier, Amsterdam, 357p.

Martel, A.E. (1971). "Coordination Chemistry". Vannostrans Reinhold, New York., 1p.

Nicholls, D. (1973). "The Chemistry of Iron, Cobalt and Nickel". Pergamon press, Oxford,1st ed.,1037, 1087, 1088,1090, 1091,1093, 1151,1154.

Pradhan, N.; Katz, B.; Efrima, S. (2003). Synthesis of high - quality metal sulfide nanoparticles from alkyl xanthate single precursors in alkylamine solvents. J. Phys. Chem. B., 107(50), $13843-13854$

Raya, I., Baba, I.; Yamin, B.M. (2006). New mixed ligand complexes of samarium(III) with dithiocarbamates and 1,10-phenanthroline. Malaysian. J. Analy. Sci., 10(1), 93-98.

Serrano, J.L.; Garc1'a, L.; Pe'rez, J.; Pe'rez, E.; Sa'nchez, G.; Garc1'a, J.; Lo'pez, G.; Garc1'a, G.; Molins, E. (2003). New dithiocarbamate and xanthate complexes of nickel(II) with iminophosphines. Inorg. Chim. Acta., 355, 33-40.

Siddiqi, K.S.; Nishat, N. (2000). Synthesis and characterization of succinimide and phthalimide dithiocarbamate and their complexes with some transition metal ions. Synth. Reac. Inorg. MetOrg. Chem., 30(8), $1505-1518$.

Singh, N.; Singh, N.K.; Kaw, C. (1989). Synthetic and spectroscopic studies of xanthate-bridged heterobimetallic complexes containing diamagnetic and paramagnetic metal ions. Bull. Chem. Soc. (Jpn.), 62, 3328.

Tiekink, E.R.T.; Haiduc, I. (2005). Stereochemical aspects of metal xanthate complexes: molecular structures and supramolecular self-assembly. Prog. Inorg. Chem., 54, 127-319.

Vogle, A.I. (2008). "Text Book of Practical Organic Chemistry". 5th edn., Pearson Education, 793p.

Wan, D.C.; Sato, K.; Kamigaito, M.; Okamoto, Y. (2005). Xanthate-mediated radical polymerization of $\mathrm{N}$-vinylpyrrolidone in fluoroalcohols for simultaneous control of molecular weight and tacticity. Macromolecule., 38, 10397-10405. 